\title{
Temperature Dependence of Charge Carrier Generation in Organic Photovoltaics
}

\author{
Feng Gao, Wolfgang Tress, Jianpu Wang and Olle Inganäs
}

\section{Linköping University Post Print}

\section{Tweet}

N.B.: When citing this work, cite the original article.

Original Publication:

Feng Gao, Wolfgang Tress, Jianpu Wang and Olle Inganäs, Temperature Dependence of Charge Carrier Generation in Organic Photovoltaics, 2015, Physical Review Letters, (114), 12, 128701.

http://dx.doi.org/10.1103/PhysRevLett.114.128701

Copyright: American Physical Society

http://www.aps.org/

Postprint available at: Linköping University Electronic Press

http://urn.kb.se/resolve?urn=urn:nbn:se:liu:diva-117377 


\title{
Temperature Dependence of Charge Carrier Generation in Organic Photovoltaics
}

\author{
Feng Gao, ${ }^{1,2, *}$ Wolfgang Tress, ${ }^{1}$ Jianpu Wang, ${ }^{2,3, \dagger}$ and Olle Inganäs ${ }^{1}$ \\ ${ }^{1}$ Biomolecular and Organic Electronics, IFM, Linköping University, Linköping 58183, Sweden \\ ${ }^{2}$ Cavendish Laboratory, J J Thomson Avenue, Cambridge CB3 OHE, United Kingdom \\ ${ }^{3}$ Key Laboratory of Flexible Electronics (KLOFE) and Institute of Advanced Materials (IAM), \\ National Synergistic Innovation Centre for Advanced Materials (SICAM), Nanjing Tech University, \\ 30 South Puzhu Road, Nanjing 211816, China
}

(Received 26 November 2014; published 27 March 2015)

\begin{abstract}
The charge generation mechanism in organic photovoltaics is a fundamental yet heavily debated issue. All the generated charges recombine at the open-circuit voltage $\left(V_{\mathrm{OC}}\right)$, so that investigation of recombined charges at $V_{\mathrm{OC}}$ provides a unique approach to understanding charge generation. At low temperatures, we observe a decrease of $V_{\mathrm{OC}}$, which is attributed to reduced charge separation. Comparison between benchmark polymer:fullerene and polymer:polymer blends highlights the critical role of charge delocalization in charge separation and emphasizes the importance of entropy in charge generation.
\end{abstract}

DOI: 10.1103/PhysRevLett.114.128701

PACS numbers: 88.40.jr, 72.20.Jv, 73.61.Ph

The internal quantum efficiency (IQE) of organic photovoltaics (OPVs), made from a blend of nanostructured donor and acceptor components, has approached $100 \%$ in highly efficient devices [1]. Light absorption in donor or acceptor components of OPVs generates strongly bound electronhole pairs, known as excitons. These excitons dissociate at the interfaces between the donor and acceptor materials, contributing to charge carrier generation. However, the physics behind this close-to-unity exciton dissociation efficiency remains heavily debated [2-7], hampering a rational design of highly efficient donor-acceptor heterojunctions.

It has been demonstrated that excitons dissociate into free carriers via intermediate states, known as charge-transfer (CT) states [8]. There are several different models proposed to explain efficient separation of CT states, including semiclassical kinetic and thermodynamic models as well as quantum mechanical models. A key debate between the kinetic and the thermodynamic model is whether the CT state separation proceeds through excited (so-called hot) or relaxed (so-called cool) CT states [9-12]. Strong evidence for relaxed CT state separation is a constant IQE extending to the low energy regime where only direct CT photoexcitation exists [13]. However, based on the assumption of an initial electron-hole distance of 1 to $2 \mathrm{~nm}$ in CT states and hence, a Coulombic binding energy much larger than the thermal energy at room temperature (RT), it is unclear what the driving force is to efficiently split these strongly bound CT states. Some alternative explanations for efficient CT state separation include the contribution from high local mobility and disorder, which account for the cooling of charge carriers due to hopping within the disordered density of states [14-18]. Very recently, a quantum mechanical model suggests that charge delocalization might play a key role in ultrafast free charge carrier generation [19-24]. Delocalization results in a large initial electron-hole distance in highly efficient blends and hence small CT state binding energy $[20,21]$.

These recent debates, most of which are based on advanced transient absorption measurements, show that there are many open questions concerning the charge generation process in OPVs. Conflicting conclusions arise from different studies, and no consensus has been reached for this fundamental issue yet. Temperature could potentially provide valuable information to understand this issue from a new perspective [7]. However, the effect of temperature on charge generation is barely investigated, presumably due to the fact that several other processes, like charge transport, also significantly depend on temperature, complicating the analysis.

In this Letter, we investigate the effect of temperature $(T)$ on charge generation by examining the open-circuit voltage $\left(V_{\mathrm{OC}}\right)$ in a wide temperature range (30 to $290 \mathrm{~K}$ ). Since all the generated charge carriers recombine at $V_{\mathrm{OC}}$, the investigation of recombined charge carriers at $V_{\mathrm{OC}}$ provides a unique approach to understanding charge generation. In addition, no long-range charge transport issue is involved at $V_{\mathrm{OC}}$, enabling us to exclusively focus on the charge separation process. At low $T$, we observe a decrease of $V_{\mathrm{OC}}$, which is rationalized in terms of reduced charge separation. Our results support the model which emphasizes the importance of charge delocalization on charge separation and also highlight the critical role of entropy in helping charge generation.

Assuming quasiequilibrium and neglecting losses at the contacts, $V_{\mathrm{OC}}$ of OPVs is determined by quasi-Fermi level difference between electrons and holes, and can be quantified as [25]

$$
e V_{\mathrm{OC}}=E_{F}^{n}-E_{F}^{p}=E_{g}-k_{B} T \ln \frac{N_{C} N_{V}}{n p},
$$


where $e$ is the elementary charge, $E_{F}^{n}\left(E_{F}^{p}\right)$ is the quasi-Fermi level for electrons (holes), $E_{g}$ is the effective band gap of the blend, $k_{B}$ is the Boltzmann constant, $n(p)$ is the free electron (hole) carrier density, and $N_{C}\left(N_{V}\right)$ is the effective density of states for electrons (holes). Unlike crystalline semiconductors, $N_{C}$ and $N_{V}$ for organic semiconductors have to be seen as more general parameters, which describe occupation and availability of states for electrons and holes. Nevertheless, most critical is the dependence of $V_{\mathrm{OC}}$ on charge carrier density $(n p)$ related to the chemical potential, independent of the exact expressions for $N_{C}\left(N_{V}\right)$. A rough examination of Eq. (1) predicts a linear increase of $V_{\mathrm{OC}}$ with decreasing temperature, which has been reported in previous publications, especially at relatively high $T$ (usually above $150 \mathrm{~K}$ ) [26-28]. However, several reports found that the situation becomes complicated at low $T$, demonstrating a sublinear relation in the $V_{\mathrm{OC}}-T$ plot with $V_{\mathrm{OC}}$ saturating and even decreasing at low $T$ [29-31].

We measured temperature-dependent $V_{\mathrm{OC}}$ for the benchmark P3HT: $\mathrm{PC}_{60} \mathrm{BM}$ blends (see the inset of Fig. 1 for chemical structures) [32] and also extended the measurements to polymer:polymer blends, which have attracted significant attention recently due to much improved performance [33]. We employed P3HT:F8TBT as an example for polymer:polymer blends. By keeping the same donor (P3HT), the comparison between $\mathrm{P} 3 \mathrm{HT}: \mathrm{PC}_{60} \mathrm{BM}$ and P3HT:F8TBT enables us to compare the effects of fullerene and polymer acceptors. In Fig. 1(a), we plot $V_{\mathrm{OC}}$ values of different devices in a wide temperature range. Consistent with previous reports in $\mathrm{P} 3 \mathrm{HT}: \mathrm{PC}_{60} \mathrm{BM}$ devices, we observe a sublinear relation in the $V_{\mathrm{OC}}-T$ plot at low $T$
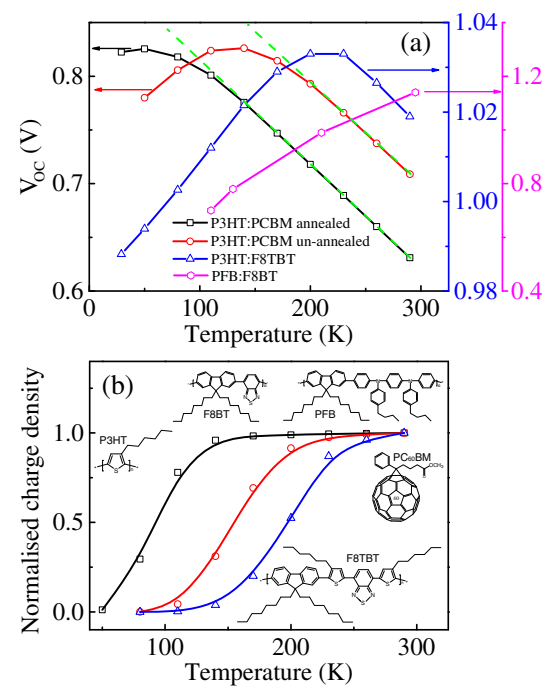

FIG. 1 (color online). (a) Temperature-dependent $V_{\mathrm{OC}}$ for P3HT:PC 60 BM, P3HT:F8TBT, and PFB:F8BT devices (chemical structures in the inset of Panel b). At low $T$, the $V_{\mathrm{OC}}-T$ plot deviates from the linear relation (dashed line), starting to saturate and even decrease. We attribute this sublinear $V_{\mathrm{OC}}-T$ relation to temperature-dependent charge separation. (b) Calculated temperature-dependent charge densities. The solid lines are guides for the eye. Panel (b) shares the same legend as Panel (a).
$[29,30]$. As indicated by the dashed lines in the figure, the annealed P3HT: $\mathrm{PC}_{60} \mathrm{BM}$ device shows an obvious deviation from the linear part at $\sim 110 \mathrm{~K}$, and the deviation point increases to $170 \mathrm{~K}$ for the unannealed device. In addition, at low $T$, we observe a decrease of $V_{\mathrm{OC}}$ for the unannealed P3HT: $\mathrm{PC}_{60} \mathrm{BM}$ device, starting from $140 \mathrm{~K}$. For the P3HT:F8TBT device, $V_{\mathrm{OC}}$ starts to decrease from $230 \mathrm{~K}$. Note that we can exclude the possibility that the decrease of $V_{\mathrm{OC}}$ is caused by the leakage current at low $T$, because the leakage current is significantly low compared with the photocurrent (Fig. S3). In Fig. 1(a), we also include the $V_{\mathrm{OC}}-T$ curve for the PFB:F8BT blend, with the data extracted from Ref. [34]. PFB:F8BT is a well-studied polymer:polymer blend with the external quantum efficiency (EQE) of $\sim 6 \%$, which is much lower than that of the P3HT:F8TBT device ( 25\%) [19]. $V_{\text {OC }}$ of the PFB:F8BT device keeps decreasing from RT to low $T$.

A careful examination of Eq. (1) reveals several possibilities that could potentially result in the sublinear relation in the $V_{\mathrm{OC}}-T$ plot in Fig. 1(a). Firstly, Eq. (1) assumes selective contacts between the electrodes and the active layer. In the case of nonselective contacts, the built-in potential is the only driving force for charges to the respective contacts, meaning that the maximum $V_{\mathrm{OC}}$ is limited by the built-in potential. We employ drift-diffusion simulations to examine whether this limit will result in a sublinear $V_{\mathrm{OC}}-T$ plot. As shown in Fig. S1, although the limit due to the built-in potential might explain the saturation of $V_{\mathrm{OC}}$ at low $T$, it cannot explain the decrease of $V_{\text {OC }}$.

Secondly, disorder, which is intrinsic to organic semiconductors, is not considered in Eq. (1) [15]. In order to examine whether the effect of disorder could be strong enough to cause a decrease of $V_{\mathrm{OC}}$ with decreasing temperature, we calculate $V_{\mathrm{OC}}$ with the aid of Fermi-Dirac statistics and a Gaussian density of states. As shown in Fig. S2, $V_{\mathrm{OC}}$ follows a nonlinear relation with $T$ and tends to saturate for low $T$ due to the occupation of the density of states which is increasingly restricted to the tail. However, this disorder effect cannot result in a decrease of $V_{\mathrm{OC}}$ with decreasing temperature.

Thirdly, the effective band gap of the blend can decrease at low $T$. This effect, seen by a slight decrease of the energy of the CT state $\left(E_{\mathrm{CT}}\right)$ [26], is weak and hence, not enough to explain the decreased $V_{\mathrm{OC}}$ in the experiment [35]. In addition, $E_{\mathrm{CT}}(\mathrm{T})$ is linear [26], which is unlikely to result in a saturation of $V_{\mathrm{OC}}$ according to Eq. (1).

Having excluded all these possibilities, we consider the last one: the decreased $V_{\mathrm{OC}}$ results from decreased charge carrier densities $(n p)$ with decreasing temperature. This means that charge separation is dependent on $T$. Based on Eq. (1), we can calculate how the charge carrier densities change with temperature. $E_{g}$ is obtained by extrapolating the linear part of the $V_{\mathrm{OC}}-T$ curve to $0 \mathrm{~K}$. Consistent with previous measurements of the CT state emission, the $E_{g}$ of annealed P3HT: $\mathrm{PC}_{60} \mathrm{BM}$ devices is smaller than that of the unannealed devices [36]. For the P3HT:F8TBT device, it is 
difficult to obtain a linear part, and the first two data points are hence used to estimate the $E_{g}$, which could be underestimated to some extent. The calculated normalized carrier densities are presented in Fig. 1(b). For the annealed P3HT: $\mathrm{PC}_{60} \mathrm{BM}$ device, the charge density remains constant from RT down to $140 \mathrm{~K}$, meaning that charge separation is efficient in a wide temperature range, agreeing well with previous transient microwave conductivity measurements [37]. Below $110 \mathrm{~K}$, the carrier density starts to decrease, meaning that charge separation becomes difficult at cryogenic temperatures. For the unannealed device, charge carrier density starts to decrease at relatively high temperatures, indicating less efficient charge separation. For the P3HT:F8TBT device, the charge carrier density already decreases from RT, meaning that charge separation limits $V_{\mathrm{OC}}$ even at RT [38].

Complementary information on the temperature dependence of charge separation is obtained from temperaturedependent $\mathrm{EQE}$ values measured at low excitation intensity. At low intensities, the recombination between free charge carriers (bimolecular recombination) is very small, as there is little chance for them to meet. At high intensities (Fig. S3), temperature dependence of short-circuit current will is stronger due to more bimolecular recombination [39]. Therefore, the EQE loss at low intensities is mainly due to charge separation. We plot the EQE values against $T$ for different devices in Fig. 2. The temperature-dependent EQE values for all the devices can be fitted using the following equation

$$
\mathrm{EQE}=\mathrm{EQE}_{0} \exp \left(-\frac{E_{a}}{k_{B} T}\right)
$$

where $\mathrm{EQE}_{0}$ is the $\mathrm{EQE}$ value at infinite temperature, and $E_{a}$ is the activation energy. The devices share a similar $\mathrm{EQE}_{0}$ value of $(85 \pm 5) \%$. The difference between $\mathrm{EQE}_{0}$ and unity is due to absorption loss. The fitted $E_{a}$ values for PFB:F8BT, P3HT:F8TBT, unannealed and annealed P3HT: $\mathrm{PC}_{60} \mathrm{BM}$ devices are $95 \pm 2,37 \pm 2,25 \pm 2$ and $9 \pm 1 \mathrm{meV}$, respectively.

The $E_{a}$ value indicates the energy required for the geminate pair separation, provided that bimolecular recombination is negligible. We exclude the possibility that $E_{a}$

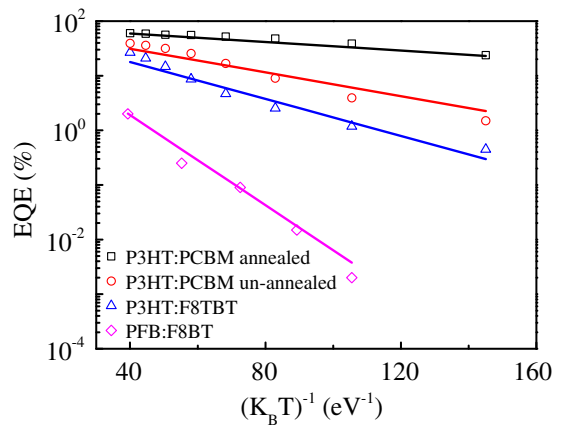

FIG. 2 (color online). Temperature-dependent EQE values at a low intensity. The points are the raw data, and the solid lines are the fitted curves (see text for details). represents the energy for thermally activated charge transport, since the activation energy for transport has been demonstrated to be one order of magnitude larger than the $E_{a}$ values here [28]. We understand that there might be some bimolecular recombination at low $T$, even at low excitation intensities. Neglecting bimolecular recombination makes us overestimate $E_{a}$. In addition, $E_{a}$ values are fitted using the $\mathrm{EQE}$ values under short-circuit conditions, where the internal field already helps geminate pair separation, especially at low $T$ [39]. This makes us underestimate $E_{a}$.

In spite of these approximations, the striking difference between $E_{a}$ for different blends does indicate that charge separation becomes increasingly difficult from the P3HT: $\mathrm{PC}_{60} \mathrm{BM}$ to the PFB:F8BT device. The trend in $E_{a}$ is consistent with our interpretation of the sublinear $V_{\mathrm{OC}}-T$ plot using temperature-dependent charge separation. The $E_{a}$ value for the annealed P3HT: $\mathrm{PC}_{60} \mathrm{BM}$ device is around $9 \mathrm{meV}$, which corresponds to the thermal energy at about $100 \mathrm{~K}$, implying that geminate recombination is dominant below $100 \mathrm{~K}$. This agrees well with Fig. 1(b), which shows that carrier density keeps constant from $290 \mathrm{~K}$ to $140 \mathrm{~K}$ and starts to decrease quickly below $110 \mathrm{~K}$. For both polymer:polymer blends, $E_{a}$ values are larger than the thermal energy at RT $(25 \mathrm{meV})$. Therefore, geminate recombination is responsible for a large fraction of EQE loss even at RT, consistent with our conclusion drawn from $V_{\mathrm{OC}}-T$ measurements as well as previous spectroscopic investigations [19].

We have demonstrated that charge separation is dependent on temperature. We now turn to the physical reasons behind it. A straightforward reason for decreased charge separation would be decreased electron-hole distance in CT states at low $T$. However, it has been demonstrated that this distance is independent of temperature by transient absorption as well as electron spin resonance susceptibility measurements $[20,40]$. For example, in P3HT: $\mathrm{PC}_{60} \mathrm{BM}$ blend, this distance has been demonstrated to be $\sim 4 \mathrm{~nm}$ $[20,21]$, corresponding to a binding energy of $\sim 100 \mathrm{meV}$, which is much larger than the thermal energy at RT. A further consideration is to take the effect of entropy $(S)$ into account. It is the free energy $(G)$, which includes both enthalpy $(H)$ and entropy, that determines whether holes and electrons are free from each other $[3,41]$. Since there is no change in pressure or volume during charge separation process, enthalpy is determined by potential energy $(U)$. Therefore,

$G=H-T S=U-T S=-\frac{e}{4 \pi \varepsilon_{0} \varepsilon_{r} r}-k_{B} T \ln (W)$,

where $\varepsilon_{0}$ is the vacuum permittivity, $\varepsilon_{r}$ is the relative dielectric constant of the blend, $r$ is the electron-hole distance of the CT state, and $W$ is the electronic degeneracy, i.e., the molecular states available to accommodate electrons and holes. From Eq. (3), it is clear that the free energy is dependent on $T$, due to the temperature-dependent contribution from entropy. This explains temperature-dependent 


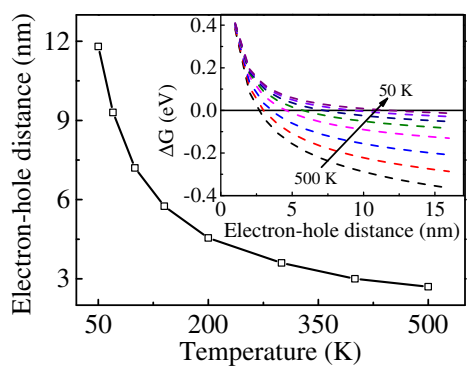

FIG. 3 (color online). Increasing electron-hole distance is required for charge separation with decreasing temperature. The inset shows the free energy dependence on the electron -hole distance at different temperatures. A negative value of $\Delta G$ means that the entropy contribution cancels the Coulombic attraction, leading to spontaneous charge separation.

charge separation, even though the initial electron-hole distance in CT states is independent of temperature.

We can further quantify the effect of temperature on charge separation, based on Eq. (3). For simplicity, holes are assumed to be localized at the interface, and only electrons can move in a hemisphere extending from the interface. Each fullerene molecule is assumed to occupy $1 \mathrm{~nm}^{3}$, and $\varepsilon_{r}$ is assumed to be 3.5. We assume that each fullerene molecule can accommodate one electron, and we understand that the exact number does not affect our qualitative analysis. The dependence of $\Delta G$ on electron-hole distance at different temperatures is shown in the inset of Fig. 3. A negative value of $\Delta G$ means that the entropy contribution cancels the Coulombic attraction, leading to spontaneous charge separation. At RT, $\Delta G$ changes sign at $\sim 4 \mathrm{~nm}$, meaning that electrons and holes are free from each other at this distance. We understand that there are several assumptions in our calculation [42]. We have underestimated the entropy by neglecting the disorder of the local energetic states. Nevertheless, this value of $\sim 4 \mathrm{~nm}$ has clearly demonstrated the importance of entropy in charge separation process. If only Coulombic attraction is considered, we usually need a puzzling value of $16 \mathrm{~nm}$ to separate electrons and holes. With decreasing temperature, $\Delta G$ changes sign at increasing distance, as summarized in Fig. 3. The reason is that the entropy contribution to free energy decreases with decreasing temperature. As a result, the geminate electron-hole pair requires a larger initial distance to separate at low $T$.

Having understood $T$-dependent charge separation, we now proceed to compare different systems. Different charge generation in different systems supports a model which emphasizes the importance of charge delocalisation in helping charge separation. The charge delocalization could be hole delocalization in the semicrystalline polymers (comparison between P3HT:F8TBT and PFB:F8BT) and/or electron delocalization in the fullerene clusters (comparison between $\mathrm{P} 3 \mathrm{HT}: \mathrm{PC}_{60} \mathrm{BM}$ and $\mathrm{P} 3 \mathrm{HT}$ : F8TBT). The delocalization distance increases from the PFB:F8BT to the P3HT: $\mathrm{PC}_{60} \mathrm{BM}$ device, resulting in higher separation efficiency for the latter.

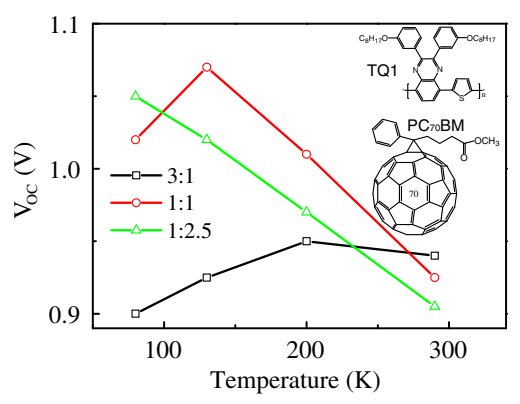

FIG. 4 (color online). Temperature-dependent $V_{\mathrm{OC}}$ for $\mathrm{TQ} 1: \mathrm{PC}_{70} \mathrm{BM}$ blends with different weight ratios. With decreasing fullerene concentration, the transition temperature, where $V_{\mathrm{OC}}$ starts to decrease, appears at higher $T$, indicating that charge separation becomes increasingly difficult with decreasing fullerene content.

In order to further demonstrate that $T$-dependent $V_{\mathrm{OC}}$ measurements provide valuable information on charge separation, we designed a straightforward experiment to measure $T$-dependent $V_{\mathrm{OC}}$ values for devices with different fullerene ratios. Gélinas et al. have recently demonstrated that the fullerene clusters play a key role in charge delocalization and charge separation [20]. Based on our previous analysis, we would expect to see different temperature dependence of $V_{\mathrm{OC}}$ for devices with different fullerene ratios, due to different charge separation efficiencies. In Fig. 4, we plot $V_{\mathrm{OC}}$ values at different temperatures for three devices with different TQ1: $\mathrm{PC}_{70} \mathrm{BM}$ weight ratios $(1: 2.5,1: 1$, and $3: 1)$ (see the inset for chemical structures). The $1: 2.5$ device gives the best performance with a power conversion efficiency of $\sim 6 \%$, and the device performance decreases with decreasing fullerene content [43]. For the 1:2.5 device, $V_{\mathrm{OC}}$ keeps increasing with decreasing temperature, while for the $1: 1$ device and $1: 3$ devices, $V_{\mathrm{OC}}$ values start to decrease at $\sim 120 \mathrm{~K}$ and $200 \mathrm{~K}$, respectively. We exclude the effect from different exciton diffusion by comparing the photoluminescence of different blends at different temperatures (Fig. S4). We can also exclude the possibility that the decrease of $V_{\mathrm{OC}}$ is caused by the leakage current at low $T$ (Fig. S5). Therefore, based on our interpretation of the $V_{\mathrm{OC}}-T$ plot, this result indicates that charge separation becomes increasingly difficult with decreasing fullerene content (and hence, smaller delocalization distance), consistent with recent spectroscopic measurements [20].

In conclusion, we employ straightforward temperaturedependent $V_{\mathrm{OC}}$ measurements to tackle a complicated and fundamental issue in OPVs, i.e., charge generation. We observe a decrease of $V_{\mathrm{OC}}$ at low $T$, resulting in a maximum in the $V_{\mathrm{OC}^{-}} T$ plot. This behavior is rationalized in terms of reduced charge separation. We find that the temperature, at which $V_{\mathrm{OC}}$ is maximum, correlates with the nanocrystallinity in the active layer, yielding an activation energy of $9 \mathrm{meV}$ for charge separation in ordered P3HT: $\mathrm{PC}_{60} \mathrm{BM}$ blend and over $25 \mathrm{meV}$ in less-ordered polymer:polymer blend. 
We thank Professor Neil C. Greenham and Professor Koen Vandewal for helpful discussions, and Dr. Ergang Wang for providing TQ1. We are grateful to the National Basic Research Program of China (Grant No. 2015CB932200), Natural Science Foundation of Jiangsu (Grant No. BK20131413), National Natural Science Foundation of China (Grant No. 11474164), the Swedish Research Council (VR), the European Commission Marie Skłodowska-Curie actions, the Swedish Energy Agency, and the Knut and Alice Wallenberg foundation (KAW) for financial support.

* Corresponding author.

fenga@ifm.liu.se

Corresponding author. iamjpwang@njut.edu.cn

[1] G. Li, R. Zhu, and Y. Yang, Nat. Photonics 6, 153 (2012).

[2] V. D. Mihailetchi, L. J. A. Koster, J. C. Hummelen, and P. W. M. Blom, Phys. Rev. Lett. 93, 216601 (2004).

[3] T. M. Clarke and J. R. Durrant, Chem. Rev. 110, 6736 (2010).

[4] J.-L. Brédas, J. E. Norton, J. Cornil, and V. Coropceanu, Acc. Chem. Res. 42, 1691 (2009).

[5] C. Deibel, T. Strobel, and V. Dyakonov, Phys. Rev. Lett. 103, 036402 (2009).

[6] R. A. J. Janssen and J. Nelson, Adv. Mater. 25, 1847 (2013).

[7] F. Gao and O. Inganäs, Phys. Chem. Chem. Phys. 16, 20291 (2014).

[8] K. Vandewal, K. Tvingstedt, A. Gadisa, O. Inganäs, and J. V. Manca, Nat. Mater. 8, 904 (2009).

[9] H. Ohkita, S. Cook, Y. Astuti, W. Duffy, S. Tierney, W. Zhang, M. Heeney, I. McCulloch, J. Nelson, D. D. C. Bradley, and J. R. Durrant, J. Am. Chem. Soc. 130, 3030 (2008).

[10] T. M. Clarke, A. M. Ballantyne, J. Nelson, D. D. C. Bradley, and J. R. Durrant, Adv. Funct. Mater. 18, 4029 (2008).

[11] G. Grancini, M. Maiuri, D. Fazzi, A. Petrozza, H.-J. Egelhaaf, D. Brida, G. Cerullo, and G. Lanzani, Nat. Mater. 12, 29 (2013).

[12] A. E. Jailaubekov, A. P. Willard, J. R. Tritsch, W.-L. Chan, N. Sai, R. Gearba, L. G. Kaake, K. J. Williams, K. Leung, P. J. Rossky, and X.-Y. Zhu, Nat. Mater. 12, 66 (2013).

[13] K. Vandewal, S. Albrecht, E. T. Hoke, K. R. Graham, J. Widmer, J. D. Douglas, M. Schubert, W. R. Mateker, J. T. Bloking, G. F. Burkhard, A. Sellinger, J. M. J. Fréchet, A. Amassian, M. K. Riede, M. D. McGehee, D. Neher, and A. Salleo, Nat. Mater. 13, 63 (2014).

[14] D. Veldman, O. İpek, S. C. J. Meskers, J. Sweelssen, M. M. Koetse, S. C. Veenstra, J. M. Kroon, S. S. van Bavel, J. Loos, and R. A. J. Janssen, J. Am. Chem. Soc. 130, 7721 (2008).

[15] U. Albrecht and H. Bässler, Chem. Phys. Lett. 235, 389 (1995)

[16] T. M. Burke and M. D. McGehee, Adv. Mater. 26, 1923 (2014).

[17] D. A. Vithanage, A. Devižis, V. Abramavičius, Y. Infahsaeng, D. Abramavičius, R. C. I. MacKenzie, P. E. Keivanidis, A. Yartsev, D. Hertel, J. Nelson, V. Sundström, and V. Gulbinas, Nat. Commun. 4, 2334 (2013).

[18] A. Melianas, V. Pranculis, A. Devižis, V. Gulbinas, O. Inganäs, and M. Kemerink, Adv. Funct. Mater. 24, 4507 (2014).
[19] A. A. Bakulin, A. Rao, V. G. Pavelyev, P. H. M. van Loosdrecht, M. S. Pshenichnikov, D. Niedzialek, J. Cornil, D. Beljonne, and R. H. Friend, Science 335, 1340 (2012).

[20] S. Gélinas, A. Rao, A. Kumar, S. L. Smith, A. W. Chin, J. Clark, T. S. van der Poll, G. C. Bazan, and R. H. Friend, Science 343, 512 (2014).

[21] A. J. Barker, K. Chen, and J. M. Hodgkiss, J. Am. Chem. Soc. 136, 12018 (2014).

[22] B. M. Savoie, A. Rao, A. A. Bakulin, S. Gelinas, B. Movaghar, R. H. Friend, T. J. Marks, and M. A. Ratner, J. Am. Chem. Soc. 136, 2876 (2014).

[23] B. Bernardo, D. Cheyns, B. Verreet, R. D. Schaller, B. P. Rand, and N. C. Giebink, Nat. Commun. 5, 3245 (2014).

[24] S. M. Falke, C. A. Rozzi, D. Brida, M. Maiuri, M. Amato, E. Sommer, A. D. Sio, A. Rubio, G. Cerullo, E. Molinari, and C. Lienau, Science 344, 1001 (2014).

[25] D. Cheyns, J. Poortmans, P. Heremans, C. Deibel, S. Verlaak, B. P. Rand, and J. Genoe, Phys. Rev. B 77, 165332 (2008).

[26] K. Vandewal, K. Tvingstedt, A. Gadisa, O. Inganäs, and J. V. Manca, Phys. Rev. B 81, 125204 (2010).

[27] S. R. Cowan, A. Roy, and A. J. Heeger, Phys. Rev. B 82, 245207 (2010).

[28] Z. Liang, A. M. Nardes, J. van de Lagemaat, and B. A. Gregg, Adv. Funct. Mater. 22, 1087 (2012).

[29] D. Rauh, A. Wagenpfahl, C. Deibel, and V. Dyakonov, Appl. Phys. Lett. 98, 133301 (2011).

[30] A. K. Thakur, G. Wantz, G. Garcia-Belmonte, J. Bisquert, and L. Hirsch, Sol. Energy Mater. Sol. Cells 95, 2131 (2011).

[31] B. P. Rand, D. P. Burk, and S. R. Forrest, Phys. Rev. B 75, 115327 (2007).

[32] See Supplemental Material at http://link.aps.org/ supplemental/10.1103/PhysRevLett.114.128701 for experimental methods, the effect of built-in voltage on Voc, the effect of disorder on Voc, the dark current at different temperatures and photoluminescence at different temperatures.

[33] A. Facchetti, Mater. Today 16, 123 (2013).

[34] H. Clubb, Ph.D. thesis, University of Cambridge, 2009.

[35] M. O. Osotov, V. V. Bruevich, and D. Y. Paraschuk, J. Chem. Phys. 131, 094906 (2009).

[36] K. Tvingstedt, K. Vandewal, A. Gadisa, F. Zhang, J. Manca, and O. Inganäs, J. Am. Chem. Soc. 131, 11819 (2009).

[37] D. H. K. Murthy, M. Gao, M. J. W. Vermeulen, L. D. A. Siebbeles, and T. J. Savenije, J. Phys. Chem. C 116, 9214 (2012).

[38] C. R. McNeill, A. Abrusci, I. Hwang, M. A. Ruderer, P. Müller-Buschbaum, and N.C. Greenham, Adv. Funct. Mater. 19, 3103 (2009).

[39] F. Gao, J. Wang, J. C. Blakesley, I. Hwang, Z. Li, and N. C. Greenham, Adv. Energy Mater. 2, 956 (2012).

[40] A. D. Chepelianskii, J. Wang, and R. H. Friend, Phys. Rev. Lett. 112, 126802 (2014).

[41] P. K. Nayak, K. L. Narasimhan, and D. Cahen, J. Phys. Chem. Lett. 4, 1707 (2013).

[42] B. A. Gregg, J. Phys. Chem. Lett. 2, 3013 (2011).

[43] E. Wang, L. Hou, Z. Wang, S. Hellström, F. Zhang, O. Inganäs, and M. R. Andersson, Adv. Mater. 22, 5240 (2010). 